contrast is typically observed as an increase in responding in the unchanged component during the transition from MULT VI VI to MULT VI EXT (Reynolds, 1961). Response rates in the VI component of the rats trained on MULT VI EXT was not higher than the VI rates observed in the other groups of rats. Behavioral contrast would not, however, be expected under these conditions, since the removal of reinforcer delivery in the dim component was accomplished at the beginning of the experiment and not after exposure to a positive reinforcement schedule in that component.

\section{REFERENCES}

Appel, J. B., \& Hiss, R. H. The discrimination of contingent and noncontingent reinforcement. Journal of Comparative \& Physiological Psychology, 1962, 55, 37-39.

Hernstein, R. J. Superstition: A corollary of the principles of operant conditioning. In W. K. Honig (Ed.), Operant behavior: Areas of research and application. New York:
Appleton-Century-Crofts, 1966. Pp. 33-51.

Lattal, K. A., \& Maxey, G. C. Some effects of response independent reinforcers in multiple schedules. Journal of the Experimental Analysis of Behavior, 1971, 16, 225-331.

Neuringer, A. J. Superstitious key pecking after three peck-produced reinforcements. Journal of the Experimental Analy sis of Behavior, 1970, 13, 127-134.

Rescorla, R. A., \& Skucy, J. C. Effect of response-independent reinforcers during extinction. Journal of Comparative \& Physiological Psychology, 1969, 67, 381-389.

Reynolds, G. S. Behavioral contrast. Journal of the Experimental Analy sis of Behavior, 1961, 4, 55-71.

Reynolds, G. S. Induction, contrast, and resistance to extinction. Journal of the Experimental Analysis of Behavior, 1968, 11, 453-457.

Skinner, B. F. "Superstition" in the pigeon. Journal of Experimental Psychology, 1948, 38, 168-172.

Zeiler, M. D. Fixed variable schedules of response-independent reinforcement. Journal of the Experimental Analysis of Behavior, 1968, 11, 405-414.

(Received for publication January 7, 1974.)

\title{
Inferences and predictions: Normative vs representative responding
}

\author{
R. JAMES HOLZWORTH* and MICHAEL E. DOHERTY \\ Bowling Green State University, Bowling Green, Ohio 43403
}

\begin{abstract}
Ss in an inference task were given sequences of data in a symmetric, binary bookbag-and-poker-chip task. They responded not only with subjective probability estimates, but also which hypothesis they considered favored. Given the same sequences in a prediction task, the same Ss made predictions of the next-to-be-observed datum. For the latter task, differential outcomes are expected under the normative Bayesian model and the representativeness heuristic. The representativeness heuristic, which leads to the expectation that Ss' predictions would often run counter to the evidence, was supported.
\end{abstract}

The hypothesis that man "relies on a limited number of heuristics which sometimes yield reasonable judgments and sometimes lead to severe and systematic errors [Kahneman \& Tversky, 1973]" is in clear contradistinction to the hypothesis that man can be described as a "conservative" Bayesian (Peterson \& Beach, 1967). The latter hypothesis has come under frequent attack as failing to account for experimental data (e.g., Bauer, 1973; Shanteau, 1970; Slovic \& Lichtenstein, 1971; Steinmann \& Doherty, 1972), and the "representativeness heuristic" (Kahneman \& Tversky, 1972, 1973; Tversky \& Kahneman, 1971), may under certain circumstances provide explicit, a priori

*Mail requests for reprints to: $\mathbf{R}$. James Holzworth, Department of Psychology, Bowling Green State University, Bowling Green, Ohio 43403. predictions which are in opposition to normative Bayesian predictions.

Representativeness is the postulated tendency for Ss in a judgment task to evaluate "the probability of an uncertain event, or a sample, by the degree to which it is: (1) similar in essential properties to its parent population; and (2) reflects the salient features of the process by which it is generated [Kahneman \& Tversky, 1972, p. 431]." Both the Bayesian and representativeness approaches predict the same outcome for the usual bookbag-and-poker-chip study, which we will term an inference procedure. In a typical inference procedure, two potential data sources, or hypotheses, would be specified in terms of prior probability, data would be presented favoring one hypothesis, and $\mathrm{S}$ would be required to state the posterior probability of 
Table 1

The 16 Stimulus Sequences Used in Each of the Two Conditions (90-10 Condition, 70-30 Condition)

\begin{tabular}{ll}
\hline 90-10 Condition & $70-30$ Condition \\
\hline WWWWWWWWW & BWBBWWWBW \\
BWBWBBBBB & WBBWWWBBB \\
BBBBBBBBB & BWWWBBBBB \\
WWWBWWWWW & WWBBWBWWB \\
BWWWWWWWW & WBWBWWWBW \\
WWWWWWWWW & BBBWWWBBB \\
WWWWWWWWW & WWWBWBWWW \\
BBBBBBBBB & BWBBBWWBB \\
BBBBBBBBB & BBWBWBWWW \\
WWWBWBWWW & BBWWBWWWW \\
BBWBBBBBB & BWWBBWBWW \\
BWWBWWWWW & WBWBBBBBW \\
BWBBBBWBB & WWBBBBBBB \\
BBBBBBBBB & BBWBBWWBW \\
BBBBBBBBB & BBWWBBBBW \\
WWWWWBWWW & WBWWWWWBW \\
\hline
\end{tabular}

Note-B denotes BLACK stimulus card, $W$ denotes WHITE stimulus card.

the source favored by the data. If, however, the $\mathrm{S}$ is asked to make a prediction about which datum will be observed on the next trial, and to state its probability, the outcomes expected under Bayesian and representativeness hypotheses are no longer the same. Such a task will be referred to as a prediction task. Given data in favor of one data source, the Bayesian $S$ would always state a probability in excess of .5 that the next datum would also favor that source. Conversely, an S behaving according to the representativeness heuristic would be expected to predict that the next datum would be such as to bring the sample proportion back into line with the population proportion, as $S$ understood it. Thus, in a bookbag-and-poker-chip prediction task, one would expect a negative recency effect according to the representativeness heuristic (Kahneman \& Tversky, 1972 , p. 435), but not according to the Bayesian hypothesis. Brickman and Pierce (1972) interpreted a negative recency effect for a task similar to a prediction task in terms of representativeness. Since their Ss were instructed as to which data source to assume and which datum to predict, Ss should have given the same response on every trial. Those authors pointed out that this may have placed unusual demand characteristics on the Ss.

The present study compares Ss' behavior in an inference task and a prediction task, both of which require Ss to make both categorical and probabilistic responses.

\section{METHOD}

\section{Subjects}

Thirty-two university students received $\$ 5$ each for serving in two 1-h sessions.

\section{Stimulus Materinl}

Each trial, or sequence, had nine IBM computer cards on which either BLACK or WHITE was printed. There were 16 sequences used in each of two conditions, with a card at the beginning of each sequence instructing $S$ that a new sequence was to begin. These sequences were generated randomly with the restriction that the population proportions given Ss were approximated by the total numbers of BLACK or WHITE cards $\mathrm{S}$ saw in the condition; they are presented in Table 1 . The cards were contained in a card holder such that a single stimulus could be withdrawn while the remaining stimuli remained out of sight.

The sequences were drawn from one of two possible populations, or decks of cards, the compositions of which were given to $\mathrm{S}$ and displayed prominently on a card during the experiment.

\section{Design}

Each $\mathrm{S}$ served in two tasks, inference and prediction, under one of two deck compositions (also referred to as diagnosticities, or population proportions). Ss were randomly assigned to one of the levels of diagnosticity upon entering the experiment. Half of the Ss were presented with 16 sequences drawn from a $90 \%$ BLACK-10\% WHITE deck or a $10 \%$ BLACK-90\% WHITE deck (90-10 condition). The same 16 sequences, with the order reversed such that the 16 th sequence became the first, were presented in the inference and prediction tasks. The other half of the Ss were presented with 16 sequences drawn from a $70 \%$ BLACK-30\% WHITE deck or a $30 \%$ BLACK-70\% WHITE deck (70-30 condition). These Ss received the same sequence, in reversed order, in each task. The sequences of stimuli in each task were identical for all Ss under a given condition. Half of the Ss performed the inference task in Session 1, half performed the prediction task in Session 1.

\section{Inference Task}

The inference task was similar to the one used by Phillips and Edwards (1966). The Ss were required to revise their prior probabilities as to which of two hypotheses was correct, in light of successive data. Each $S$ was presented with 16 sequences, and wrote on each card from which deck he believed the sequence had come, and his estimate of subjective probability that it had so come, $\mathbf{P}(H / D)$.

\section{Prediction Task}

The responses in the prediction task were the predicted color of the next-to-be-observed card, and the subjective estimate of that probability.

\section{Procedure}

Ss were tested individually. Each $S$ had before him a card holder containing one practice sequence, 16 experimental sequences, and a display card. Before beginning the actual experimental trials in each session, $S$ read the instructions for the appropriate task. After reading the instructions, $S$ was presented a practice sequence, and permitted to ask questions. Prior to the first observation, and after each of the nine stimuli, $S$ made either an inference or a prediction. In the prediction task, $S$ was instructed to predict what the 10th card "would have been." After each response, $S$ turned over the stimulus card and went on to the next one.

No feedback was provided as to the accuracy of the subjective probability estimates. After the nine cards in a sequence were observed, $S$ began the next sequence, unti all 16 were completed.

\section{RESULTS}

The typical presentation of the results of bookbag-and-poker-chip experiments in terms of the regression of subjective odds on Bayesian odds is obviated by large numbers of predictions of the less likely datum in the prediction task. This result is not unexpected if one regards the experiment as being 
Table 2

The Number of Counterevidentiary Responses Made Immediately Following Runs of Varying Lengths at Each Level of s-f Except Zero

\begin{tabular}{|c|c|c|c|c|c|c|c|}
\hline \multirow{2}{*}{$\begin{array}{l}\text { Run } \\
\text { Length }\end{array}$} & \multicolumn{5}{|c|}{$s-f$} & \multirow[b]{2}{*}{ Total } & \multirow[b]{2}{*}{ Proportions } \\
\hline & 1 & 2 & 3 & 4 & 5 & & \\
\hline 1 & $144 / 656$ & $37 / 176$ & $21 / 80$ & $4 / 16$ & $3 / 16$ & $179 / 944$ & .19 \\
\hline 2 & $54 / 192$ & $72 / 240$ & $12 / 32$ & $4 / 16$ & - & $142 / 480$ & .30 \\
\hline 3 & $30 / 80$ & $29 / 64$ & $51 / 112$ & - & $7 / 16$ & $117 / 272$ & .43 \\
\hline 4 & - & $16 / 32$ & $13 / 32$ & $17 / 32$ & - & $46 / 96$ & .48 \\
\hline $5-7$ & - & - & $15 / 32$ & $20 / 32$ & $13 / 32$ & $48 / 96$ & .50 \\
\hline $\begin{array}{l}\text { Total } \\
\text { Proportions }\end{array}$ & $\begin{array}{c}198 / 928 \\
.21\end{array}$ & $\begin{array}{c}154 / 512 \\
.30\end{array}$ & $\begin{array}{c}112 / 288 \\
.39\end{array}$ & $\begin{array}{c}45 / 96 \\
.47\end{array}$ & $\begin{array}{c}23 / 64 \\
.36\end{array}$ & $\begin{array}{c}532 / 1888 \\
.28\end{array}$ & .28 \\
\hline
\end{tabular}

Note-The denominator is the total number of possible responses in that cell and is a multiple of 16, since there were 16 Ss.

within a probability learning tradition, but it is totally inconsistent with a Bayesian interpretation. Fully $28.2 \%$ of the 70-30 prediction responses were counter to the evidence; the percentages for the 70-30 inference, $90-10$ prediction and 90-10 inference tasks being negligible (3.9, 3.4, and 0.8). Another aspect of nonoptimal responding in the prediction condition is shown by the percentage of subjective probabilities exceeding the population proportion-31.5\% and $15.7 \%$ in the $70-30$ and 90-10 conditions, respectively. The results for the 70-30 prediction condition will be explored in detail.

The counterevidentiary revisions are expected under the representativeness heuristic, which implies that Ss should show an increasing proportion of such revisions as a function of several indices of sample composition. Table 2 shows the proportions of counterevidentiary revisions as a function of run length, and as a function of the typical Bayesian parameter, s-f. Furthermore, if one makes the simple assumption, as strongly implied by Kahneman and Tversky (1972), that Ss given $\mathrm{P}(\mathrm{H})$ would probability-match in their predictions, then one can make explicit predictions of when Ss should make a counterevidentiary response. Simply put, all predictions should be such as to bring the sample proportion closer to the population proportion. Given this assumption, and given the sequences shown in Table 1, 45 of the 144 predictions each $S$ made should have been counterevidentiary. Of these, averaging across all Ss, $37.8 \%$ were in the direction predicted by the representativeness heuristic. Only $16.4 \%$ of the responses not predicted to be counterevidentiary were so.

\section{DISCUSSION}

The inference condition of the present study was like the typical Bayesian study, except that Ss made a categorical choice as well as stating a probability value. The prediction condition was like a typical probability learning experiment, except that Ss were given the prior probabilities and population proportions to start with. The gambler's fallacy or negative recency effect was very strong in the 70-30 prediction condition, as expected under representativeness. Its virtual absence in the $90-10$ prediction condition, in spite of the long runs and high s-f values, indicate strongly that Ss were highly sensitive to the differential proportions of white and black cards in the two populations, as given them in the instructions.
The Ss' sensitivity to population proportions is contrary to what Kahneman and Tversky (1972) found in the symmetric binomial problem, but is actually more in accord with the concept of representativeness than the data they explored. Certainly the population proportion is an "essential feature of the population," which the sample should reflect. The discrepancy between their results and the ones reported here is superficial, owing to their having discarded Ss who made counterevidentiary responses, whereas such responses were the primary data of the present analysis.

The relative absence of counterevidentiary responses in the 70-30 inference conditions, which had exactly the same sequence of stimuli, and in the 90-10 conditions which had longer runs, shows clearly that these responses are not just some automatic response to the stimulus sequences, but are under the control of cognitive factors. It must be noted, however, that the Ss were not in any sense behaving completely representatively. The counterevidentiary predictions are so egregiously irrational, from a normative standpoint, that they compel the attention; but two-thirds of those responses expected to be counterevidentiary were not. On balance, however, the data presented are critical of the normative model qua descriptive model; and, more clearly than expected, positively supportive of the representativeness hypothesis.

\section{REFERENCES}

Bauer, M. Subjective representation and prediction in probabilistic inference tasks. Project Report, Cognitive Processes Unit, Department of Psychology, University of Umeå, March 1973.

Brickman, P., \& Pierce, S. M. Estimates of conditional probabilities of confirming versus disconfirming events as a function of inference situation and prior evidence. Journal of Experim ental Psychology, 1972, 95, 235-237.

Kahneman, D., \& Tversky, A. Subjective probability: A judgment of representativeness. Cognitive Psychology, 1972, 3, 430-454.

Kahneman, D.. \& Tversky, A. On the psychology of prediction. Psychological Review, 1973, 4, 237-251.

Peterson, C. R., \& Beach, L. R. Man as an intuitive statistician. Psychological Bulletin, 1967, 68, 29-46.

Phillips, L. D., \& Edwards, W. Conservatism in a simple probability inference task. Journal of Experimental Psychology, 1966, 72, 346-354.

Slovic, P., \& Lichtenstein, S. Comparison of Bayesian and regression approaches to the study of information processing in judgment. Organizational Behavior \& Human Performance, $1971,6,649-744$.

Steinmann, D. O., \& Doherty, M. E. A lens model analysis of a bookbag-and-poker-chip experiment: A methodological note. Organizational Behavior \& Human Performance, 1972, 8, 450-455.

Tversky, A., \& Kahneman, D. Belief in the new law of small numbers. Psychological Bulletin, 1971, 76, 105-110.

Shanteau, J. C. An additive model for decision-making. Journal of Experimental Psychology, 1970, 85, 181-191.

(Received for publication January 22, 1974.) 